\title{
CORRECCIÓN DE LA ACIDEZ DEL SUELO: ALTERACIONES QUÍMICAS DEL SUELO
}

\author{
Siavosh Sadeghian Khalajabadi (D) *, Carolina Díaz Marín (D) **
}

\begin{abstract}
Sadeghian-Khalajabadi, S., \& Díaz-Marín, C. (2020). Corrección de la acidez del suelo: Alteraciones químicas del suelo. Revista Cenicafé, 71(1), 7-20. https://doi.org/10.38141/10778/1116
\end{abstract}

\begin{abstract}
La alternativa más común para corregir la acidez del suelo consiste en el empleo de enmiendas calcáreas. El objetivo de esta investigación fue determinar el efecto del encalamiento en la fertilidad de algunos suelos representativos de la zona cafetera de Colombia. Se evaluaron las propiedades químicas de cinco suelos luego de un mes de incubación con cinco dosis de cal $\left(0,00 ; 1,25 ; 2,50 ; 5,00\right.$ y $\left.10,00 \mathrm{~g} \mathrm{dm}^{3}\right)$. Los tratamientos afectaron diferencialmente a los suelos en su $\mathrm{pH}, \mathrm{Ca}^{2+}, \mathrm{Mg}^{2+}, \mathrm{CIC}, \mathrm{Al}^{3+}, \mathrm{Fe}^{2+}, \mathrm{Mn}^{2+}, \mathrm{NO}_{3}^{-}$, saturación de bases y relación de bases intercambiables. El pH del suelo logró explicar con mayor ajuste las variaciones de $\mathrm{Al}^{3+}$ que el porcentaje de saturación de $\mathrm{Al}^{3+}$. La CIC, determinada mediante el acetato de amonio $1 \mathrm{M}$ y con $\mathrm{pH}=7,0$, no fue un método adecuado para determinar el efecto del encalamiento; caso contrario ocurrió con la solución no-tamponada de cloruro de bario. Se presentó una alta correlación entre el porcentaje de saturación de bases y el pH para cada suelo; sin embargo, no se encontró una tendencia que pudiera explicar las variaciones de todos los suelos de manera conjunta. Los cambios de la CIC, generados por el incremento del $\mathrm{pH}$ que ocasionó el encalamiento, estuvieron en función de los contenidos de la materia orgánica. Una parte de los contenidos de $\mathrm{Ca}^{2+} \mathrm{y} \mathrm{Mg}^{2+}$ determinados en el análisis del suelo correspondió a la fracción no intercambiable, resultado que se relacionó con la baja solubilidad de la cal y soporta su efecto residual.
\end{abstract}

Palabras clave: Encalado, fertilidad del suelo, aluminio, bases intercambiables, CIC.

\section{SOIL ACIDITY CORRECTION: SOIL CHEMICAL ALTERATIONS}

The most common alternative to correct soil acidity consists of using calcareous amendments. The objective of this work of research was to determine the effect of liming on the fertility of some soils representative of the Colombia coffee region. The chemical properties of five soils were evaluated after one month of incubation with five doses of lime $\left(0.00,1.25,2.50,5.00\right.$ and $\left.10.00 \mathrm{~g} \mathrm{dm}^{3}\right)$. The treatments differentially affected the soils in their $\mathrm{pH}, \mathrm{Ca}^{2+}, \mathrm{Mg}^{2+}, \mathrm{CEC}, \mathrm{Al}^{3+}, \mathrm{Fe}^{2+}, \mathrm{Mn}^{2+}, \mathrm{NO}^{3-}$, base saturation and exchangeable base ratio. The $\mathrm{pH}$ of the soil evidenced the $\mathrm{Al}^{3+}$ variations more considerably than the saturation percentage of $\mathrm{Al}^{3+}$. CEC, determined by $1 \mathrm{M}$ ammonium acetate and with $\mathrm{pH}=7.0$, was not a good method to estimate the effect of liming, whereas the non-buffered solution of barium chloride showed to be effective. There was a high correlation between the base saturation percentage and the $\mathrm{pH}$ for each soil. However, it was not possible to find a trend to explain the variations of all soils together. The changes of CEC, generated by the $\mathrm{pH}$ increase caused by liming, were in accordance the organic matter contents. A part of the contents of $\mathrm{Ca}^{2+}$ and $\mathrm{Mg}^{2+}$, determined in the soil analysis, corresponded to the non-exchangeable fraction, a result that was related to the low solubility of the lime and supports its residual effect

Keywords: Liming, soil fertility, aluminum, exchangeable bases, CEC.

\footnotetext{
* Investigador Científico III. Disciplina de Suelos, Centro Nacional de Investigaciones de Café, Cenicafé. Chinchiná, Caldas, Colombia. https://orcid.org/0000-0003-1266-0885.

** Ingeniera agrónoma, M.Sc. https://orcid.org/0000-0002-6843-7503.
} 
En muchas regiones de Colombia la acidez del suelo constituye una limitante potencial para la producción de café (Sadeghian, 2013). Aunque esta condición ocurre de manera natural, principalmente como consecuencia del material parental del suelo en interacción con el clima y los organismos, su magnitud se intensifica en los suelos cultivados, debido a la remoción de las bases intercambiables, calcio $\left(\mathrm{Ca}^{2+}\right)$, magnesio $\left(\mathrm{Mg}^{2+}\right)$ y potasio $\left(\mathrm{K}^{+}\right)$, a través de las cosechas, la erosión, el uso de los fertilizantes amoniacales, la oxidación de la materia orgánica (MO) y la aplicación de azufre elemental (S) (Sousa et al., 2007; Zapata, 2004). Los suelos ácidos contienen cantidades relativamente altas de aluminio $\left(\mathrm{Al}^{3+}\right) \mathrm{y}$, a veces, de manganeso $\left(\mathrm{Mn}^{2+}\right)$, que resultan tóxicas para las plantas; en contraste, son pobres en $\mathrm{Ca}^{2+} \mathrm{y} \mathrm{Mg}^{2+}$ (Espinosa \& Molina, 1999; Havlin et al., 2014; Raij, 2011).

La alternativa más común para corregir la acidez del suelo radica en el empleo de materiales encalantes; práctica que eleva el $\mathrm{pH}$, neutraliza el $\mathrm{Al}^{3+} \mathrm{y} \mathrm{Mn}^{2+}$, proporciona $\mathrm{Ca}^{2+} \mathrm{y}$ $\mathrm{Mg}^{2+}$, aumenta la disponibilidad de fósforo (P) y molibdeno (Mo), favorece la mineralización de la MO, ayuda a la fijación simbiótica de nitrógeno $(\mathrm{N})$, propicia la floculación de los coloides, estimula el crecimiento radical y, por ende, mejora el aprovechamiento por parte de las plantas del agua y de los nutrientes (Raij, 2011). En suelos de carga variable, carga dependiente del $\mathrm{pH}$, el encalado incrementa la Capacidad de Intercambio Catiónico (CIC), dando como resultado una mayor retención de bases intercambiables. Los anteriores efectos han sido corroborados en investigaciones desarrolladas tanto en Colombia (Sadeghian, 2016; Suárez \& Rodríguez, 1956; Valencia \& Bravo, 1981; Vinasco et al., 1992), como otros países productores de café (Chaves et al., 1984; Chaves et al., 1988; Corrêa et al., 2007; Navarro y Navarro, 2013; Rodrigues et al., 2006).
Otro aspecto comúnmente considerado en la aplicación de las enmiendas para la corrección de la acidez tiene que ver con las relaciones entre las bases intercambiables $(\mathrm{Ca}: \mathrm{Mg}, \mathrm{Ca}: \mathrm{Ko}$ $\mathrm{Mg}: \mathrm{K})$, ello debido al antagonismo que puede existir entre estos, para ser absorbidos por las raíces de las plantas. En las investigaciones pioneras acerca del tema sobresale el estudio desarrollado por Bear y Toth (1948), quienes concluyen que idealmente un suelo debe contener $65 \%$ de $\mathrm{Ca}^{2+}, 10 \%$ de $\mathrm{Mg}^{2+}$ y $5 \%$ de $\mathrm{K}^{+}$. La validez de los anteriores criterios ha sido objeto de muchas investigaciones, y aunque algunos autores los consideran pertinentes para café (Corrêa et al., 2007; Garcia, 1981; Malavolta, 2006), en la mayoría de los casos su implementación no resulta posible en razón de las diferencias que se presentan tanto los suelos en sus propiedades, como las enmiendas y los abonos en su solubilidad y efecto residual (Järvan, 2004).

En razón de que para las condiciones de la zona cafetera de Colombia se cuenta con poca información acerca del tema, en esta investigación se planteó como objetivo, determinar el efecto del encalamiento en las propiedades de suelos.

\section{MATERIALES Y MÉTODOS}

En cinco localidades de la zona cafetera de Colombia, con diferentes grados de acidez ( $\mathrm{pH}$ entre 4,4 y 5,1), se recolectaron muestras de suelo a $20 \mathrm{~cm}$ de profundidad: i) finca cafetera en el municipio de Jamundí (Valle del Cauca); ii) Estación Experimental (EE) Naranjal (Chinchiná, Caldas); iii) EE Paraguaicito (Buenavista, Quindío); iv) EE El Rosario (Venecia, Antioquia) y v) EE San Antonio (Floridablanca, Santander). Las muestras se llevaron al Centro Nacional de Investigaciones de Café, Cenicafé, ubicado en el municipio de Manizales (Caldas, Colombia), a $5^{\circ} 1$, latitud Norte y $75^{\circ} 35^{\prime}$ longitud Oeste, con 
$1.310 \mathrm{~m}$ de altitud, temperatura promedio de $21,7^{\circ} \mathrm{C}$ y precipitación anual de $2.600 \mathrm{~mm}$.

Las muestras se homogeneizaron mediante volteos y se pasaron por zaranda de $1,0 \mathrm{~cm}$, con el fin de eliminar los terrones grandes. Aproximadamente $500 \mathrm{~g}$ de cada suelo se secaron al aire y se analizaron las siguientes propiedades, de acuerdo a los procedimientos descritos por Carrillo (1985): $\mathrm{pH}, \mathrm{MO}, \mathrm{P}, \mathrm{K}^{+}$, $\mathrm{Ca}^{2+} \mathrm{y} \mathrm{Mg}^{2+}, \mathrm{Al}^{3+}, \mathrm{Fe}, \mathrm{Mn}, \mathrm{CIC}$ y textura (Tabla 1).

Se evaluaron cinco tratamientos, constituidos por cuatro dosis de cal $(1,25 ; 2,50 ; 5,00 \mathrm{y}$ $10,00 \mathrm{~g} \mathrm{dm}^{3}$ ) y un testigo. El material calcáreo empleado para la corrección de la acidez contenía $46,6 \%$ de $\mathrm{CaO}$ y $8,1 \%$ de $\mathrm{MgO}$. Cada tratamiento fue aplicado a $30 \mathrm{dm}^{3}$ de suelo contenidos en un recipiente plástico y se incubó por 30 días, con humedad a capacidad de campo (agua retenida a presiones entre 0,033 y 1,5 MPa), tiempo después del cual se tomó una muestra para el análisis de las propiedades químicas antes mencionadas, según el protocolo de Carrillo (1985). Adicionalmente, se evaluó la concentración de nitrato $\left(\mathrm{NO}_{3}{ }^{-}\right)$ con el kit RQ-Flex y la CIC mediante los siguientes métodos: i) saturación con acetato de amonio $\left(\mathrm{CH}_{3} \mathrm{COONH}_{4}\right)$ 1,0 $\mathrm{M}$ a pH=7,0 y desplazamiento con cloruro de sodio $(\mathrm{NaCl})$ 1,0 M (Carrillo, 1985), y ii) saturación con una solución no tamponada de cloruro de bario $\left(\mathrm{BaCl}_{2}\right)$ 0,1 $\mathrm{M}+$ cloruro de amonio) $\left(\mathrm{NH}_{4} \mathrm{C} 1\right)$ 0,1 M y desplazamiento con sulfato de magnesio $\left(\mathrm{MgSO}_{4}\right)$ 0,005M (Gillman, 1979).

Se realizaron análisis de varianza por localidad $(\alpha=95 \%)$ a través del programa estadístico SAS (Statistical Analysis System), versión 9,2 de 2009, y se empleó la regresión lineal (programa Sigmaplot 10,0) con el fin de explicar los cambios de las variables. Se seleccionaron los modelos con mayor coeficiente de determinación $\left(\mathrm{R}^{2}\right)$, verificando la significancia de los parámetros de las ecuaciones.

\section{RESULTADOS Y DISCUSIÓN}

La acidez de los suelos, en sus componentes de $\mathrm{pH}$ y $\mathrm{Al}^{3+}$, se neutralizó de conformidad con las dosis de la cal, presentando algunas variaciones entre las localidades (Figura 1). Para los suelos de El Rosario, Paraguaicito y Jamundí, el incremento del pH ocurrió de manera lineal, en tanto que para Naranjal y San Antonio tuvo una tendencia de tipo exponencial asintótico (Figura 1a). El mayor incremento del $\mathrm{pH}$, calculado por la diferencia entre el tratamiento sin encalar y la dosis más alta de cal, se registró para Jamundí $(2,06)$, mientras que los menores aumentos correspondieron a Paraguaicito $(1,10)$ y El Rosario $(1,18)$; en cuanto a Naranjal y San Antonio, las diferencias entre los valores mínimos y máximos tuvieron un comportamiento intermedio $(1,74$ y 1,68, respectivamente). Era de esperarse que la aplicación de enmiendas calcáreas en suelos ácidos contribuyera a incrementar los valores de esta propiedad; de allí que los resultados obtenidos confirman los reportes de otras investigaciones (Chaves et al., 1984; Chaves et al., 1988; Corrêa et al., 2007; Pavan \& Bingham, 1982; Valencia \& Bravo, 1981; Vileta et al., 2010).

Una mayor resistencia al cambio en el pH, detectado para el suelo de El Rosario, puede atribuirse a una mayor CIC y los altos contenidos de la $\mathrm{MO}$ y de $\mathrm{Al}^{3+}$ (Tabla 1). De acuerdo con Raij (2011), la diferencia que exhiben los suelos en dicha resistencia, poder tampón o búfer, se debe a la CIC; siendo mayor esta propiedad en suelos más ricos en MO y arcilla. Para la zona cafetera de Colombia, diferentes reportes corroboran la relación de la CIC en función de la MO (Sadeghian, 2010; Sadeghian \& Zapata, 2012). También debe resaltarse que 


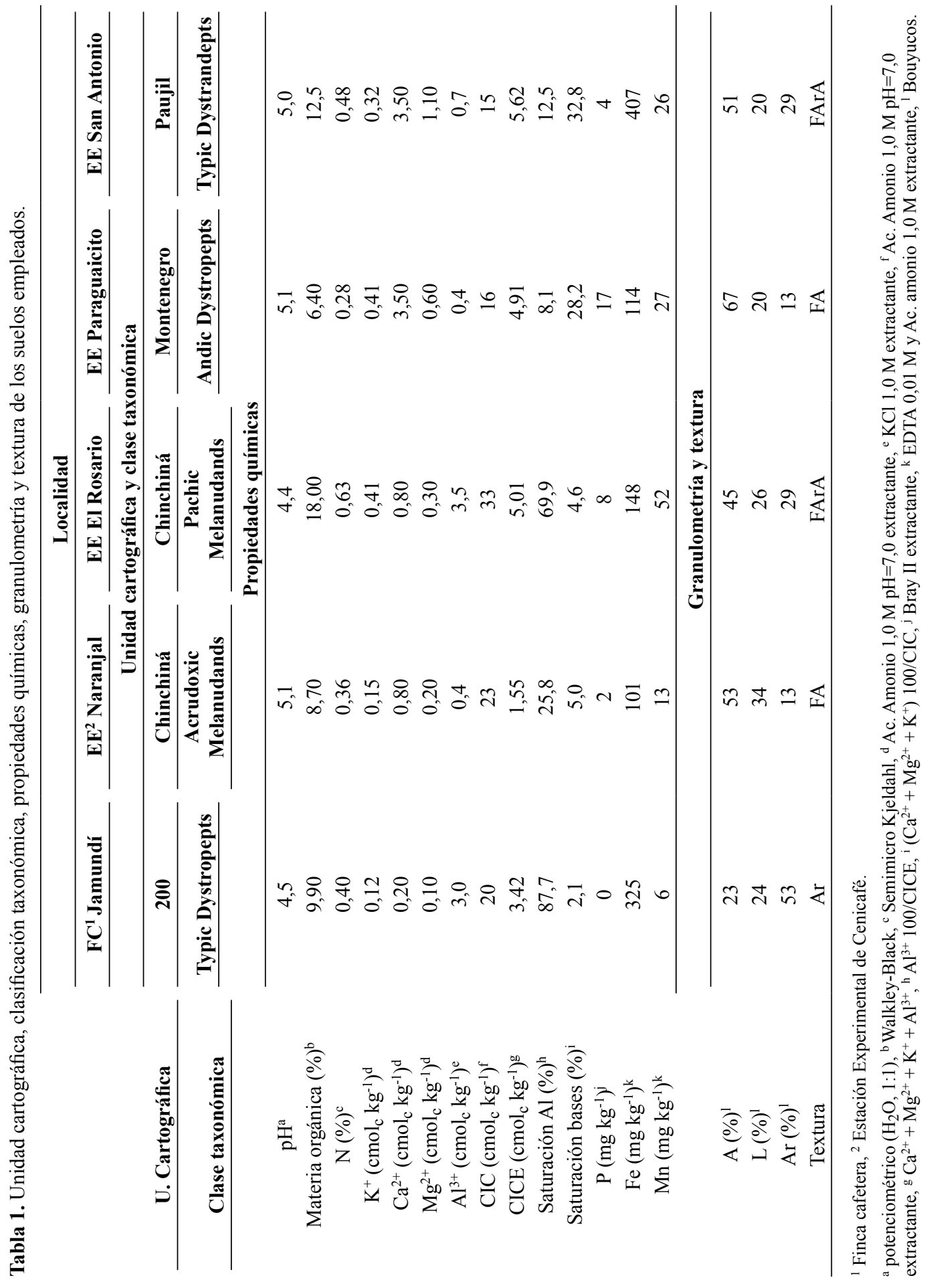




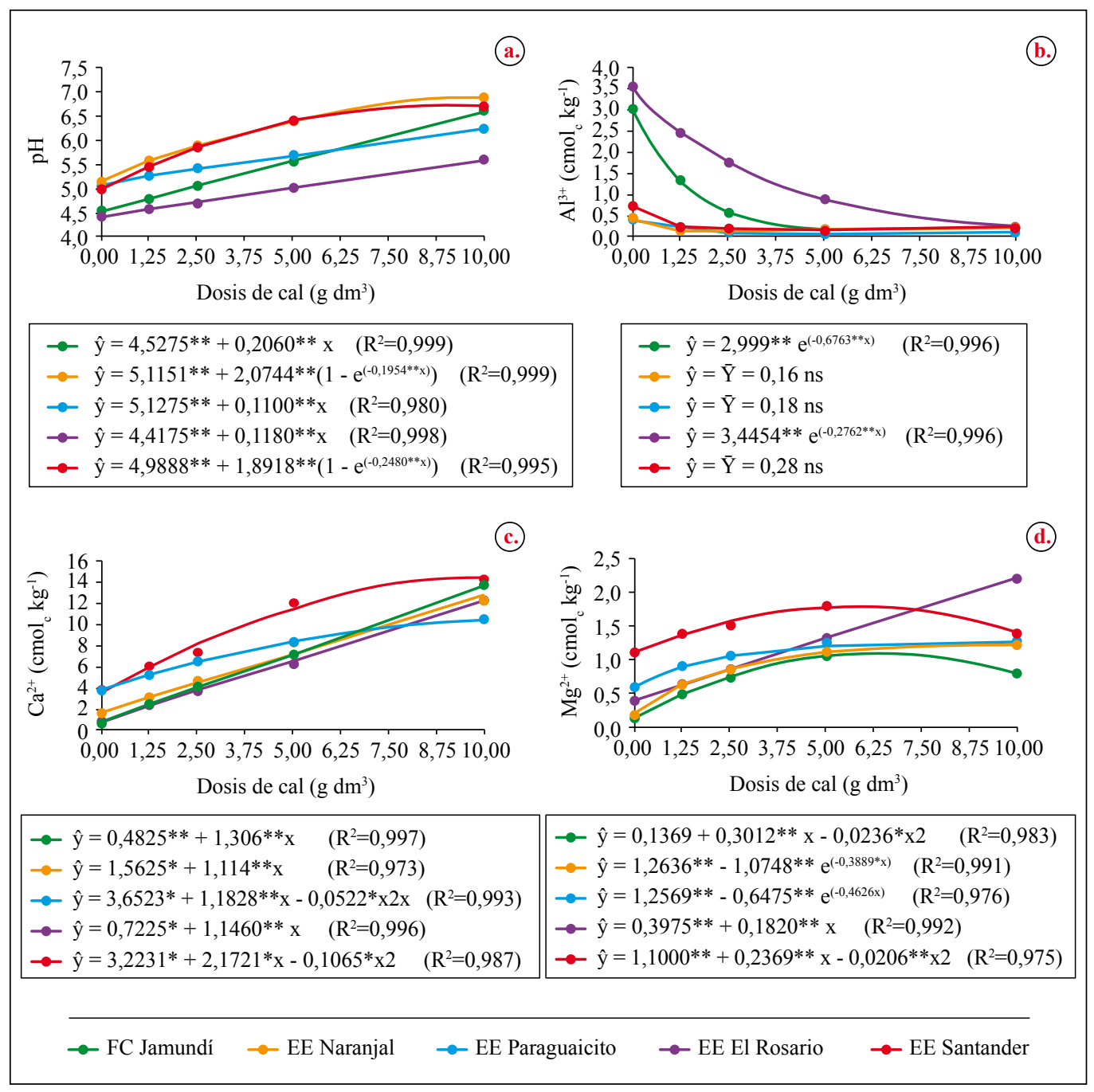

Figura 1. Variaciones del $\mathrm{pH}(\mathrm{a}), \mathrm{Al}^{3+}(\mathrm{b}), \mathrm{Ca}^{2+}(\mathrm{c}) \mathrm{y} \mathrm{Mg}^{2+}$ (d) en respuesta a las dosis de cal. ns: no significativo. * significativo $(\mathrm{p}<0,05), * *$ altamente significativo $(\mathrm{p}<0,01)$.

la elevada capacidad tampón de los Andisoles ha sido relacionada con la superficie muy reactiva de las arcillas de estos suelos, alófana, imogolita y complejos humus-Al, resultantes de la meteorización de las cenizas volcánicas (Espinosa \& Molina, 1999). Con respecto al suelo de Paraguaicito, por su textura más arenosa y menor contenido de MO, se hubieran esperado mayores incrementos del pH a dosis más bajas de cal, por su reducido poder relativo de amortiguación, como lo señalan Havlin et al. (2014). El incremento limitado del $\mathrm{pH}$ en este suelo, posiblemente estaría relacionado con el lavado del material encalante en conformidad a los riegos, una menor retención de humedad, necesaria para la reacción de la cal, y la presencia de la alófana. 
La adición de cal disminuyó de manera significativa el $\mathrm{Al}^{3+}$ en los suelos de Jamundí y El Rosario, mientras que para los demás suelos no se detectaron cambios estadísticamente significativos (Figura 1b); lo anterior debido a sus bajos contenidos del elemento (Tabla 1). Esta reducción se justifica por la producción de complejos hidroxi-aluminios, hasta llegar a la forma $\mathrm{Al}(\mathrm{OH})_{3}$; especie química insoluble que tiende a precipitar cuando el $\mathrm{pH}$ alcanza valores cercanos a la neutralidad (Sousa et al., 2007). La efectividad del encalamiento para la neutralización del $\mathrm{Al}^{3+}$ en suelos ácidos destinados al cultivo de café ha sido corroborada en otras investigaciones realizadas por Chaves et al. (1988), Pavan y Bingham (1982), Rodrigues et al. (2006), Sadeghian (2016) y Valencia (1981).

Las variaciones del $\mathrm{Al}^{3+}$ en función de las dosis de la cal fueron explicadas mediante el modelo exponencial (Figura 1b); lo cual indica que las primeras tres dosis de la enmienda (hasta $5 \mathrm{~g} \mathrm{dm}^{3}$ ) tuvieron un efecto mayor en la neutralización de este, que la última (10 g $\mathrm{dm}^{3}$ ). Similares resultados han sido obtenidos por Rodrigues et al. (2001), Rodrigues et al. (2006) y Sadeghian (2003; 2013). Cabe resaltar que para el suelo de Jamundí la tasa de la disminución de $\mathrm{Al}^{3+}$ fue mayor que el suelo de El Rosario; alcanzando de esta manera valores cercanos a cero con cantidades menores, 5, $0 \mathrm{~g}$ $\mathrm{dm}^{3}$ de cal frente a $10,0 \mathrm{~g} \mathrm{dm}^{3}$. Esta diferencia puede atribuirse al aumento del $\mathrm{pH}$ registrado en el suelo de Jamundí y menor capacidad búfer, determinada por los contenidos más bajos de MO y de CIC.

Los contenidos de $\mathrm{Ca}^{2+}$ se incrementaron con las dosis suministradas (Figura 1c), lo cual justifica el empleo de materiales encalates como fuente de este elemento, además de la corrección de la acidez. Para los suelos de Jamundí y las Estaciones Naranjal y El Rosario el aumento de $\mathrm{Ca}^{2+}$ fue proporcional a las cantidades aplicadas (tendencia lineal), en tanto que para San Antonio y Paraguaicito el comportamiento fue decreciente (tendencia cuadrática). El suelo de Paraguaicito exhibió el menor incremento de este elemento, pasando de 3,5 a $10,3 \mathrm{cmol}_{\mathrm{c}} \mathrm{kg}^{-1}$, resultado que concuerda con los cambios registrados para $\mathrm{pH}$ de esta localidad, relacionado con la capacidad de retención de humedad y la posible pérdida por lavado del material encalante. Espinosa y Molina (1999) sostienen que, en los suelos de textura liviana, la lixiviación de $\mathrm{Ca}^{2+} \mathrm{y}$ $\mathrm{Mg}^{2+}$ proveniente de la cal es mayor $\mathrm{y}$, por lo tanto, se sugiere aumentar la frecuencia del encalado.

En el suelo de El Rosario, el $\mathrm{Mg}^{2+}$ intercambiable se incrementó de manera proporcional a las dosis de cal suministradas (Figura 1d), mientras que en los demás suelos los contenidos exhibieron aumentos hasta la dosis de 5,00 $\mathrm{g} \mathrm{dm}^{3}$, para luego estabilizarse o presentar reducciones.

En otros trabajos se reportan incrementos de $\mathrm{Ca}^{2+} \mathrm{y} \mathrm{Mg}^{2+}$ al emplear la dolomita (Chaves et al., 1984; Chaves et al., 1988; Freiria et al., 2008; Pavan \& Bingham, 1982; Rodrigues et al., 2006; Valencia \& Bravo, 1981); sin embargo, es razonable pensar que no todo el $\mathrm{Ca}^{2+} \mathrm{y} \mathrm{Mg}^{2+}$ detectado en el análisis de suelo es realmente intercambiable, en especial cuando se aplicaron las dosis más altas; consideración que se soporta en la residualidad de la cal y las deficiencias de las metodologías analíticas en determinar sólo la fracción intercambiable (Soratto \& Crusciol, 2008; Raij et al., 1982).

Las diferencias entre los suelos para retener tanto el $\mathrm{Ca}^{2+}$ como $\mathrm{Mg}^{2+}$ en la fase intercambiable pueden relacionarse con diversas causas, principalmente la CIC, el $\mathrm{pH}$, la presencia de otros cationes y el tipo de arcilla, además de la selectividad de los minerales presentes por cada elemento (Bohn 
et al., 2001; Essington, 2015). En cuanto a la disminución del $\mathrm{Mg}^{2+}$ intercambiable con las dosis más altas de cal, la principal causa estaría relacionada con el aumento de la competencia ejercida por el $\mathrm{Ca}^{2+}$.

Como era de esperarse, los contenidos del $\mathrm{Al}^{3+}$ intercambiable presentaron una relación estrecha con el pH (Figura 2a), y aunque los valores de saturación de $\mathrm{Al}^{3+}$ también exhibieron una tendencia similar (Figura 2b), su correlación presentó menor ajuste, en términos de coeficiente de determinación del modelo $\left(\mathrm{R}^{2}\right)$. Los cambios observados se consideran comunes (Havlin et al., 2014; Pavan \& Bingham, 1982; Sadeghian, 2003; Sadeghian \& Zapata, 2012; Wagatsuma \& Ezoe, 1985) e indican que la magnitud en la reducción del $\mathrm{Al}^{3+}$ es mayor para valores del pH menor a 5,0. De acuerdo con los modelos obtenidos, para valores de $\mathrm{pH}$ entre 5,0 y 5,5 , rango considerado como óptimo para el café, se presentaron los siguientes intervalos de $\mathrm{Al}^{3+}$ y saturaciones de $\mathrm{Al}^{3+}: 0,75$ a 0,20 $\mathrm{cmol}_{\mathrm{c}} \mathrm{kg}^{-1}$ y 14,46 a $3,42 \%$, respectivamente.

Para cada suelo empleado el modelo lineal explicó el incremento del $\mathrm{pH}$ en función del porcentaje de saturación de bases intercambiables (Figura 3); a pesar de ello, no se encontró una tendencia común que pudiera explicar las variaciones de todos de manera conjunta; en este sentido, el suelo de Naranjal se diferenció de las demás localidades. Lo anterior sugiere que dos suelos pueden tener el mismo $\mathrm{pH}$, pero con valores diferentes de la saturación de bases. Aunque los requerimientos de la cal pueden calcularse tanto con base en el $\mathrm{pH}$ como en la saturación de bases (Quaggio et al., 1985), los valores de saturaciones encontrados para dos regiones pueden ser diferentes (Raij, 2011), afirmación que concuerda con lo hallado en este estudio. De acuerdo con Raij (2011) una saturación de bases equivalente al 50\% se alcanza cuando el $\mathrm{pH}$ del suelo es igual a 5,5; condición que no se cumplió para los suelos empleados.

El aumento en las dosis de la cal se vio reflejado en la disminución de los contenidos del Fe y parcialmente Mn (Tabla 2); entretanto, se incrementaron los niveles de los nitratos $\left(\mathrm{NO}_{3}{ }^{-}\right)$, resultado que se relaciona con el aumento en la mineralización de la MO del suelo (Chaves et al., 1988). En cuanto al P y $\mathrm{K}$, los cambios fueron leves o no ocurrieron.

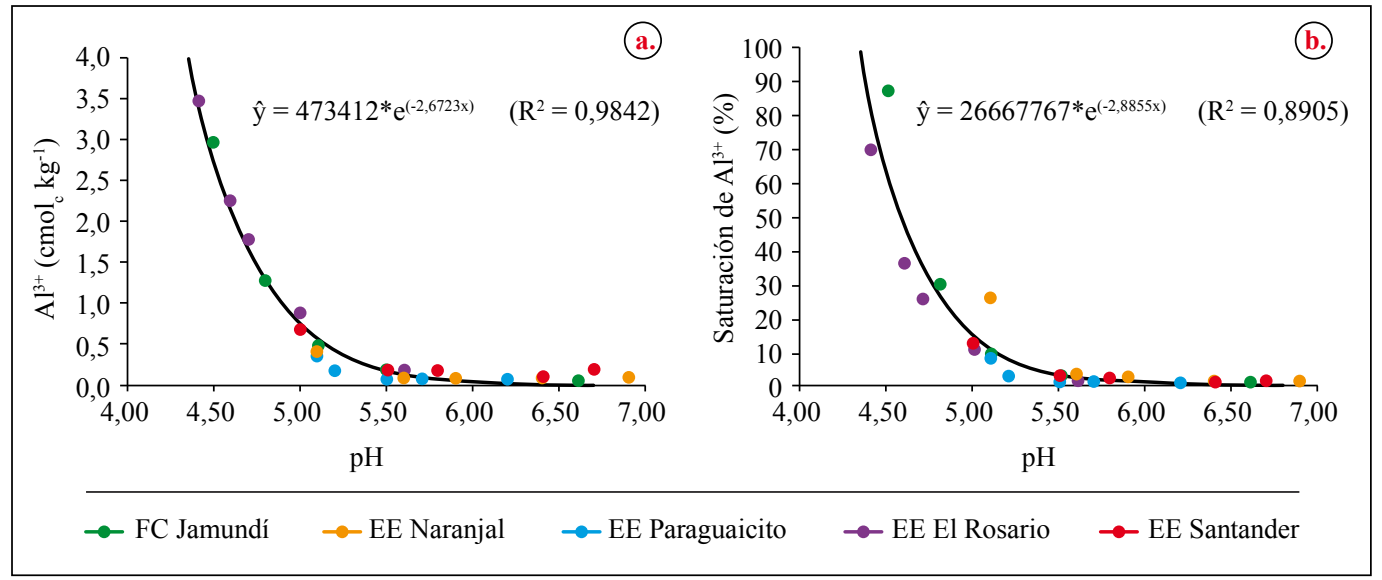

Figura 2. Variaciones del $\mathrm{Al}^{3+}$ intercambiable (a) y saturación de $\mathrm{Al}^{3+}$ (b) en función el pH. 
En investigaciones similares también hallaron evidencias del aumento en los contenidos de $\mathrm{NO}_{3}{ }^{-}$en respuesta al encalamiento (Chaves et al., 1988, Suárez \& Rodríguez, 1956) y disminución del Mn (Chaves et al., 1988; Valencia \& Bravo, 1981).

El incremento de las cantidades suministradas de cal modificó las relaciones entre las bases intercambiables ( $\mathrm{Ca}: \mathrm{Mg}, \mathrm{Ca}: \mathrm{K}$ y $\mathrm{Mg}: \mathrm{K})$, haciendo que fueran cada vez más amplias (Tabla 3). El incremento de $\mathrm{Ca}^{2+}$ con respecto al $\mathrm{Mg}^{2+}$ se debe, en primer lugar, a la preferencia de los suelos por el $\mathrm{Ca}^{2+}$, explicado por su menor radio iónico hidratado con respecto al $\mathrm{Mg}^{2+}$ (Bohn et al., 2001; Essington, 2015; Navarro \& Navarro, 2013) y, en segunda instancia, a una mayor competencia de $\mathrm{Ca}^{2+}$ al incrementar las dosis de la enmienda, la cual disminuyó la retención del $\mathrm{Mg}^{2+}$ en la fase de cambio. En cuanto al $\mathrm{K}^{+}$, era de esperarse que su proporción frente a $\mathrm{Ca}^{2+} \mathrm{y} \mathrm{Mg}^{2+}$ disminuyera, pues este no se aplicó; adicionalmente, el $\mathrm{K}^{+}$, por poseer una menor valencia es retenido con menor fuerza en los sitios de intercambio.
Corrêa et al. (2007) y Vilela et al. (2010) también registraron aumentos en la relación $\mathrm{Ca}: \mathrm{Mg}$ con la aplicación de dosis crecientes de dolomita; en contraste, en el estudio desarrollado para café por Chaves et al. (1984), se encontraron incrementos de $\mathrm{Ca}^{2+}$

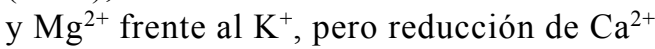
con respecto al $\mathrm{Mg}^{2+}$.

Los incrementos registrados fueron disímiles para los suelos estudiados (Tabla 3), respuesta que tiene su origen en las diferencias que exhiben en sus propiedades, especialmente la selectividad catiónica; por ejemplo, para el suelo de El Rosario, donde los aumentos del $\mathrm{Mg}^{2+}$ fueron más altos, una mayor preferencia por el catión, se presentaron los menores valores para la relación $\mathrm{Ca}: \mathrm{Mg}$. Lo anterior tiene implicaciones en los aspectos prácticos, pues al aplicar una misma dosis de enmienda se ocasionarán relaciones diferentes, según el tipo de suelo.

Para suelos ácidos y de carga variable, como los empleados en este estudio, se espera que la CIC se incremente en conformidad al

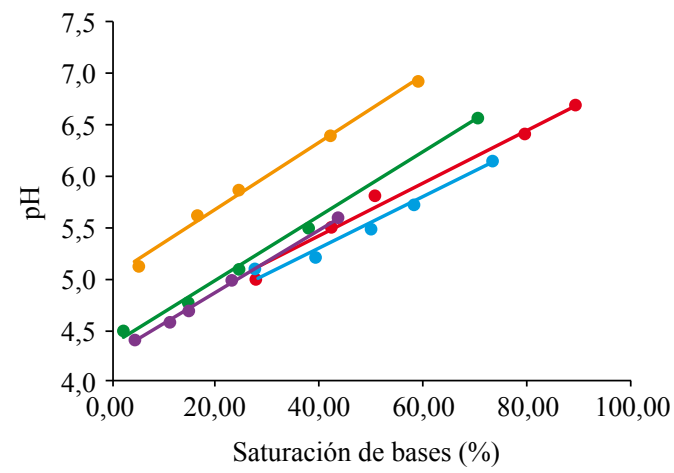

$$
\begin{array}{ll}
\multimap \hat{y}=4,3666+0,0315 x & \left(\mathrm{R}^{2}=0,996\right) \\
\multimap \hat{y}=5,0219+0,0328 x & \left(\mathrm{R}^{2}=0,991\right) \\
\multimap \hat{y}=4,3195+0,0248 x & \left(\mathrm{R}^{2}=0,961\right) \\
\multimap \hat{y}=4,2626+0,0313 x & \left(\mathrm{R}^{2}=0,998\right) \\
\multimap \hat{y}=4,3563+0,0265 x & \left(\mathrm{R}^{2}=0,989\right)
\end{array}
$$

Figura 3. Saturación de bases intercambiables en función del pH. 
aumento del $\mathrm{pH}$ que genera el encalamiento (Chaves et al., 1984, Pavan \& Bingham, 1982; Vilela et al., 2010); efecto que se relaciona con la ionización de los grupos $-\mathrm{OH}$ de las arcillas y del humus (Bohn et al., 2001; Essington, 2015; Navarro y Navarro, 2013). Al respecto, los valores de la CIC, determinados mediante el acetato de amonio (AA) con $\mathrm{pH}=7,0$, no se modificaron con los tratamientos (Figura 4a), no así los obtenidos a través de la solución no-tamponada de cloruro de bario (CB) (Figura $4 b)$. Lo anterior sugiere que la valoración de la CIC con CB puede explicar de manera más acertada el efecto de la corrección de la acidez sobre esta propiedad. Cuando se emplea el AA, el pH de la solución se eleva casi a la neutralidad, haciendo que la CIC alcance niveles cercanos al máximo posible, enmascarando así el efecto de los tratamientos del encalamiento, lo cual no sucedió con CB, dado que en este se emplea un reactivo notamponado. Fauziah et al. (1997) consideran que para los suelos ácidos del trópico la valoración de la CIC mediante el acetato de amonio tamponado $(\mathrm{pH}=7,0)$ sólo debe tenerse en cuenta para fines de clasificación, y recomiendan usar la Capacidad de Intercambio Catiónico Efectiva-CICE $\left(\mathrm{Ca}^{2+}+\mathrm{Mg}^{2+}+\right.$ $\mathrm{K}^{+}+\mathrm{Al}^{3+}$ ) en la evaluación agronómica; sugerencia que coincide con las de Silva y Mendonça (2007).

Para todos los suelos, la CIC obtenida con AA fue mayor que la determinada mediante el empleo de CB (Figura 4), aun cuando se lograron valores de $\mathrm{pH}$ cercanos a la neutralidad mediante la aplicación de la dosis más alta de cal. Para Rodrigues et al. (2001) esta situación ocurre debido a que el AA, además de elevar las cargas variables del suelo, disuelve los cationes unidos a carbonatos, los cuales en condiciones reales de campo no estarían disponibles a corto plazo para los cultivos.
El incremento de la CIC en función del $\mathrm{pH}$, medido a través del coeficiente de regresión lineal, fue mayor para el suelo de El Rosario, seguido por Jamundí, Naranjal, San Antonio y Paraguaicito (Figura $5)$, tendencia que tiene su origen en los contenidos de la MO de los suelos (Tabla 1). La naturaleza de los grupos funcionales de la MO que contribuyen a la superficie de carga dependiente del $\mathrm{pH}$ ha sido ampliamente tratada por diversos autores (Bohn et al., 2001; Essington, 2015; Navarro \& Navarro, 2013), y la relación de la CIC con la MO reportada para suelos de la zona cafetera de Colombia (Sadeghian, 2010).

Se presentó una relación lineal entre la CIC, valorada por CB, y la CICE (Figura 6). Pese a lo anterior, la ecuación de la regresión indica que los valores de la CICE fueron $62,6 \%$ mayores que los de la CIC, sugiriendo así cierta inconsistencia en la metodología analítica, pues ello indica que una fracción de las bases consideradas intercambiables no se encuentra realmente en la fase de cambio. Tratándose de tratamientos con enmiendas, pueden atribuirse las diferencias a la baja solubilidad del material encalante y su efecto residual, el cual define el momento de repetir la aplicación de la enmienda (Soratto \& Crusciol, 2008; Raij et al., 1982).

Las principales conclusiones del presente estudio son:

- El encalamiento afectó diferencialmente a los suelos en su pH, $\mathrm{Ca}^{2+}, \mathrm{Mg}^{2+}, \mathrm{CIC}, \mathrm{Al}^{3+}$, $\mathrm{Fe}^{2+}, \mathrm{Mn}^{2+}, \mathrm{NO}_{3}{ }^{-}$, saturación de bases $\mathrm{y}$ relación de bases intercambiables.

- El aumento en las cantidades suministradas de cal incrementó las relaciones $\mathrm{Ca}: \mathrm{Mg}$, $\mathrm{Ca}: \mathrm{K}$ y Mg:K.

- La CIC, determinada mediante el acetato de amonio $1 \mathrm{M}$ y con $\mathrm{pH}=7,0$, no fue un 
método adecuado para explicar el efecto del encalamiento; caso contrario ocurrió con la solución no-tamponada de cloruro de bario.

- Los cambios de la CIC, generados por el incremento del $\mathrm{pH}$ que ocasionó el encalamiento, estuvieron en función de los contenidos de la MO.
- Una parte de los contenidos de $\mathrm{Ca}$ y $\mathrm{Mg}$ determinados en el análisis del suelo correspondió a la fracción no intercambiable de estos elementos, resultado que se relacionó con la baja solubilidad de la cal y soporta su efecto residual.

Tabla 2. Cambios de $\mathrm{K}^{+}, \mathrm{P}, \mathrm{Fe}, \mathrm{Mn}$ y nitrato $\left(\mathrm{NO}_{3}{ }^{-}\right)$del suelo en respuesta a dosis de cal, aplicada en cinco suelos de la zona cafetera.

\begin{tabular}{|c|c|c|c|c|c|}
\hline $\begin{array}{c}\text { Dosis de cal } \\
\left(\mathrm{g} \mathrm{dm}^{3}\right) \\
\end{array}$ & $\begin{array}{c}\mathrm{K} \\
\left(\mathrm{cmol}_{\mathrm{c}} \mathrm{kg}^{-1}\right)\end{array}$ & $\begin{array}{c}P \\
\left(\mathrm{mg} \mathrm{kg}^{-1}\right)\end{array}$ & $\begin{array}{c}\mathrm{Fe} \\
\left(\mathrm{mg} \mathrm{kg}^{-1}\right)\end{array}$ & $\begin{array}{c}\text { Mn } \\
\left(\mathrm{mg} \mathrm{kg}^{-1}\right)\end{array}$ & $\begin{array}{c}\mathrm{NO}_{3}^{-} \\
\left(\mathrm{mg} \mathrm{kg}^{-1}\right)\end{array}$ \\
\hline \multicolumn{6}{|c|}{ FC Jamundí } \\
\hline 0,00 & 0,12 & 0 & 325 & 6 & 0 \\
\hline 1,25 & 0,20 & 1 & 323 & 6 & 15 \\
\hline 2,50 & 0,31 & 1 & 260 & 7 & 10 \\
\hline 5,00 & 0,20 & 1 & 247 & 7 & 12 \\
\hline 10,00 & 0,22 & 2 & 147 & 7 & 31 \\
\hline \multicolumn{6}{|c|}{ EE Naranjal } \\
\hline 0,00 & 0,15 & 2 & 101 & 13 & 22 \\
\hline 1,25 & 0,17 & 2 & 104 & 13 & 23 \\
\hline 2,50 & 0,20 & 3 & 90 & 11 & 28 \\
\hline 5,00 & 0,18 & 3 & 79 & 10 & 42 \\
\hline 10,00 & 0,18 & 2 & 58 & 7 & 46 \\
\hline \multicolumn{6}{|c|}{ EE Paraguaicito } \\
\hline 0,00 & 0,41 & 17 & 114 & 27 & 18 \\
\hline 1,25 & 0,49 & 20 & 99 & 23 & 77 \\
\hline 2,50 & 0,49 & 25 & 80 & 16 & 104 \\
\hline 5,00 & 0,47 & 19 & 86 & 20 & 111 \\
\hline 10,00 & 0,44 & 17 & 67 & 15 & 136 \\
\hline \multicolumn{6}{|c|}{ EE EI Rosario } \\
\hline 0,00 & 0,41 & 8 & 148 & 52 & 90 \\
\hline 1,25 & 0,85 & 6 & 153 & 33 & 81 \\
\hline 2,50 & 0,83 & 8 & 148 & 32 & 95 \\
\hline 5,00 & 0,79 & 5 & 135 & 27 & 85 \\
\hline 10,00 & 0,78 & 3 & 98 & 20 & 78 \\
\hline \multicolumn{6}{|c|}{ EE San Antonio } \\
\hline 0,00 & 0,32 & 4 & 407 & 26 & 0 \\
\hline 1,25 & 0,40 & 2 & 387 & 21 & 0 \\
\hline 2,50 & 0,40 & 2 & 341 & 21 & 11 \\
\hline 5,00 & 0,41 & 3 & 199 & 16 & 19 \\
\hline 10,00 & 0,41 & 5 & 177 & 16 & 81 \\
\hline
\end{tabular}


Tabla 3. Cambios en la relación entre cationes intercambiables del suelo en función de las dosis de cal.

\begin{tabular}{|c|c|c|c|c|c|}
\hline $\begin{array}{c}\text { Dosis de cal } \\
\left(\mathrm{g} \mathrm{dm}^{3}\right)\end{array}$ & $\begin{array}{c}\text { FC } \\
\text { Jamundí }\end{array}$ & $\begin{array}{c}\text { EE } \\
\text { Naranjal } \\
\end{array}$ & $\begin{array}{c}\text { EE } \\
\text { Paraguaicito }\end{array}$ & $\begin{array}{c}\text { EE } \\
\text { El Rosario } \\
\end{array}$ & $\begin{array}{c}\text { EE } \\
\text { San Antonio } \\
\end{array}$ \\
\hline & \multicolumn{5}{|c|}{ (Ca:Mg) } \\
\hline 0,00 & $2,0: 1$ & $4,0: 1$ & $5,8: 1$ & $2,7: 1$ & $3,2: 1$ \\
\hline 1,25 & $4,8: 1$ & $5,0: 1$ & $5,7: 1$ & $3,4: 1$ & $4,1: 1$ \\
\hline 2,50 & $5,0: 1$ & $5,8: 1$ & $6,0: 1$ & $3,9: 1$ & $4,8: 1$ \\
\hline 5,00 & $6,7: 1$ & $6,8: 1$ & $7,3: 1$ & $4,6: 1$ & $6,7: 1$ \\
\hline \multirow[t]{2}{*}{10,00} & $17,0: 1$ & $10,1: 1$ & 7,9:1 & $5,6: 1$ & $10,1: 1$ \\
\hline & \multicolumn{5}{|c|}{$(\mathrm{Ca}: \mathrm{K})$} \\
\hline 0,00 & $1,7: 1$ & $5,3: 1$ & $8,5: 1$ & $2,0: 1$ & $10,9: 1$ \\
\hline 1,25 & $12,0: 1$ & $17,6: 1$ & $10,4: 1$ & $2,8: 1$ & $14,5: 1$ \\
\hline 2,50 & $12,9: 1$ & $23,0: 1$ & $13,5: 1$ & $4,2: 1$ & $18,0: 1$ \\
\hline 5,00 & $33,5: 1$ & $45,6: 1$ & $17,0: 1$ & $7,6: 1$ & $29,3: 1$ \\
\hline \multirow[t]{2}{*}{10,00} & $61,8: 1$ & $67,2: 1$ & $23,4: 1$ & $15,9: 1$ & $34,6: 1$ \\
\hline & \multicolumn{5}{|c|}{ (Mg:K) } \\
\hline 0,00 & $0,8: 1$ & $1,3: 1$ & $1,5: 1$ & $0,7: 1$ & $3,4: 1$ \\
\hline 1,25 & $2,5: 1$ & $3,5: 1$ & $1,8: 1$ & $0,8: 1$ & $3,5: 1$ \\
\hline 2,50 & $2,6: 1$ & $4,0: 1$ & $2,2: 1$ & $1,1: 1$ & $3,8: 1$ \\
\hline 5,00 & $5,0: 1$ & $6,7: 1$ & $2,3: 1$ & $1,6: 1$ & $4,4: 1$ \\
\hline 10,00 & $3,6: 1$ & $6,7: 1$ & $3,0: 1$ & $2,8: 1$ & $3,4: 1$ \\
\hline
\end{tabular}

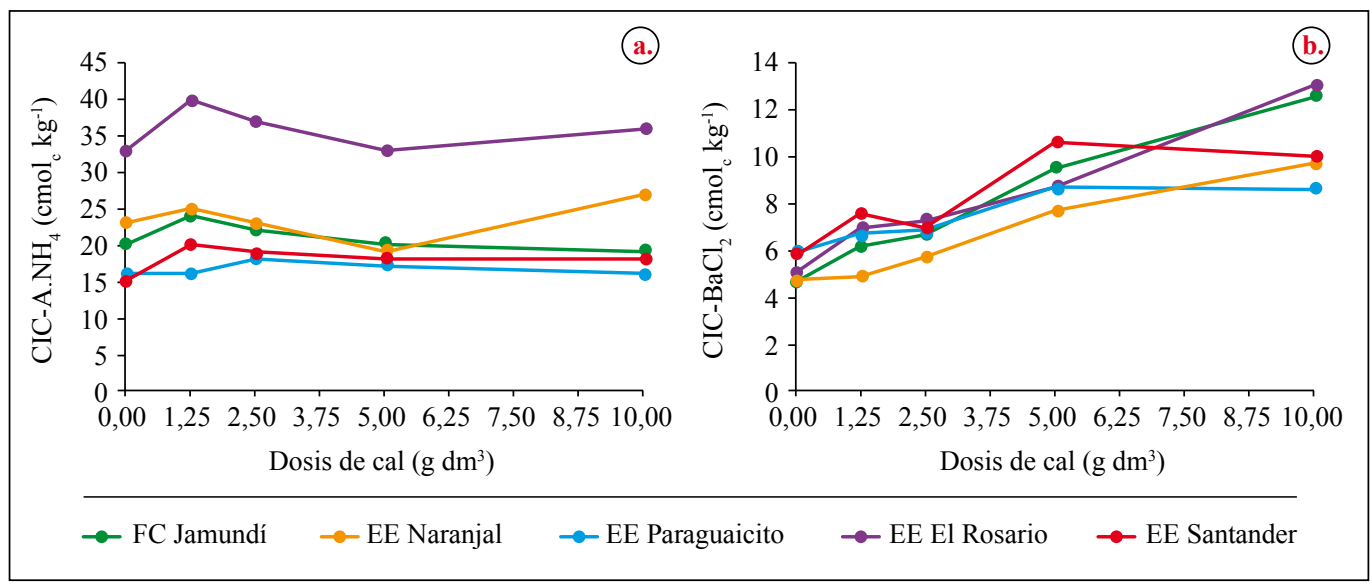

Figura 4. Cambios en la CIC, evaluada mediante acetato de amonio (a) y cloruro de bario (b), en respuesta a las dosis de cal. 


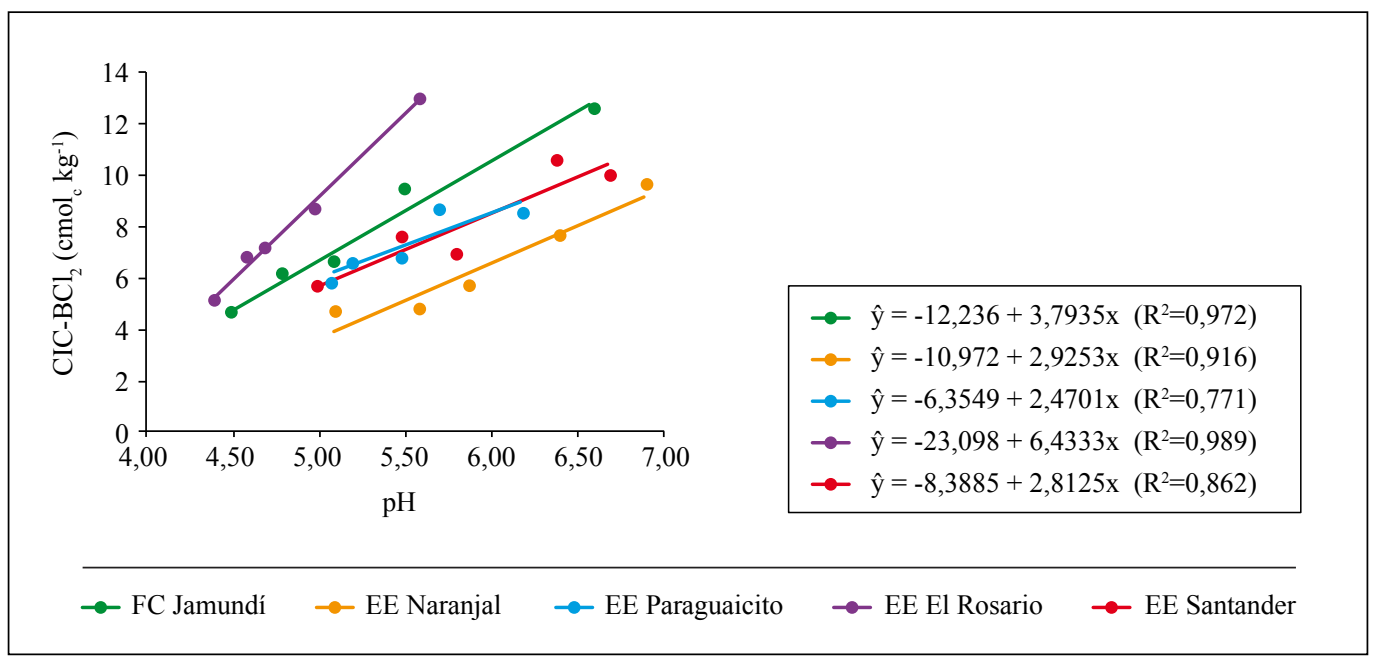

Figura 5. Variaciones de CIC (determinada por cloruro de bario), en función del pH.

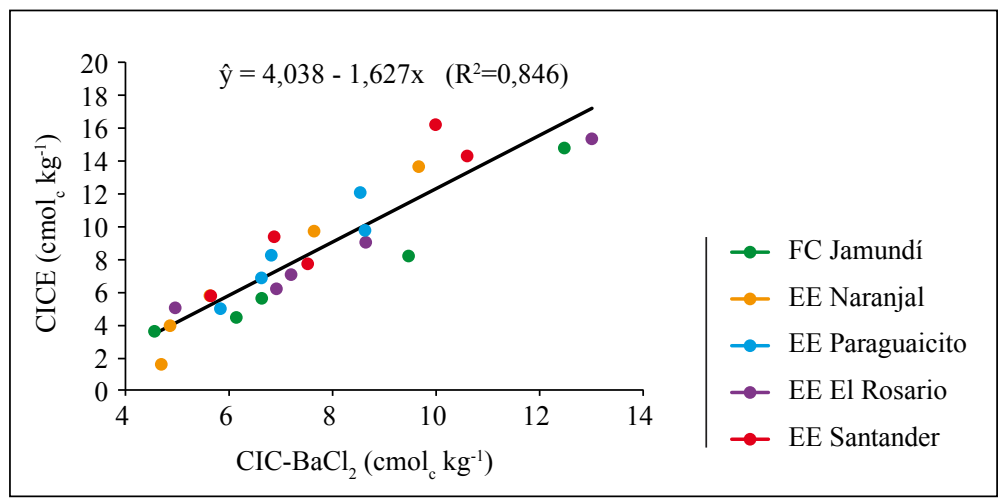

Figura 6. Relación entre CICE y CIC (determinada por cloruro de bario).

\section{LITERATURA CITADA}

Bear, F.E., \& Toth, S.J. (1948). Influence of Calcium on availability of other soil cations. Soil Science, 65(1), 69-74. https://journals.lww.com/soilsci/ citation/1948/01000/influence_of_calcium_on_ availability of other_soil.7.aspx

Bohn, H.L., McNeal, B.L., \& O’Connor, G.A. (2001). Soil Chemistry (3rd ed.). John Wiley \& Sons.

Carrillo, I. F. (1985). Manual de laboratorio de suelos. Cenicafé.
Chaves, J., Pavan, M.A., \& Igue, K. (1984). Respostas do cafeeiro à calagem. Pesquisa Agropecuária Brasileira, 19(5), 573-582. https://seer.sct.embrapa.br/index.php/ pab/article/view/15714/9728

Chaves, J., Pavan, M.A., \& Miyazawa, M. (1988). Redução da acidez subsuperficial em coluna de solo. Pesquisa Agropecuária Brasileira, 23(5), 469-476. http://seer.sct. embrapa.br/index.php/pab/article/viewFile/13891/7877

Corrêa, J.B., Reis, T.H., Pozza, A., Guimarães, P.T., \& Carvalho, J. (2007). Índice de saturação por bases na nutrição e na produtividade de cafeeiros Catuaí vermelho (Coffea arabica L.). Coffee Science, 2(2), 159-167. 
https://pdfs.semanticscholar.org/552a/13ccca2a5a05a 36e5f83a9ff4f2ed1ffla10.pdf

Espinosa, J., \& Molina, E. (1999). Acidez y encalado de los suelos. International Plant Nutrition Institute. http://www.cia.ucr.ac.cr/pdf/libros/Acidez $\% 20 \mathrm{y} \% 20$ encalado $\% 20 \mathrm{de} \% 20$ suelos, $\% 20$ libro $\% 20$ por $\% 20 \% 20$ J\%20Espinosa\%20y\%20E\%20Molina.pdf

Essington, M. (2015). Soil and water chemistry: an integrative approach (2 ed). CRC Press.

Fauziah, C.I., Jamilah, I., \& Syed, S.R. (1997). An evaluation of cation exchange capacity methods for acid tropical soils. Pertanika Journal of Tropical Agricultural Science, 20(2-3), 113-119. http://www.pertanika.upm. edu.my/Pertanika\%20PAPERS/JTAS\%20Vol.\%20 20\%20(2\&3)\%20Aug.\%201997/04\%20JTAS\%20 Vol.20\%20(23)\%201997\%20(Pg\%20115-119).pdf

Freiria, A.C., Mantovani, J. R., Ferreira, M. E., Cruz, M.C., \& Yagi, R. (2008). Alterações em atributos químicos do solo pela aplicação de calcário na superfície ou incorporado. Acta Scientiarum. Agronomy, 30(2), 285291. https://doi.org/10.4025/actasciagron.v30i2.1738

Garcia,A. (1981). Calagem para o cafeeiro. En E. Malavolta, T. Yamada \& J. Guidolin (Eds.), Nutrição e adubação do cafeeiro (pp. 103-115). Instituto da Potassa \& Fosfato e Instituto Internacional da Potassa.

Gillman, G. (1979). A proposed method for the measurement of exchange properties of highly weathered soils. Soil Research, 17(1), 129-139. https://doi.org/10.1071/ SR9790129

Havlin, J.L., Tisdale, S.L., Nelson, W.L., \& Beaton, J.D. (2014). Soil fertility and fertilizers: an introduction to nutrient management (8 ed.). Pearson.

Järvan, M. (2004). Available plant nutrients in growth substrate depending on various lime materials used for neutralising bog peat. Agronomy Research, 2(1), 29-37. https://agronomy.emu.ee/vol021/p2104.pdf

Malavolta,E.(2006). Manual de nutrição mineral de plantas. Agronômica Ceres.

Navarro, G., \& Navarro, S. (2013). Química agrícola: química del suelo y de nutrientes esenciales para las plantas. (3ra ed). Mundi-Prensa.

Pavan, M.A., \& Bingham, F.T. (1982). Toxicity of aluminum to coffee seedlings grown in nutrient solution. Soil Science Society of America Journal, 46(5), 993-997. https://doi.org/10.2136/ sssaj1982.03615995004600050021x
Quaggio, J.A., Raij, B., \& Malavolta, E. (1985). Alternative use of the SMP $\square$ buffer solution to determine lime requirement of soils. Communications in Soil Science and Plant Analysis, 16(3), 245-260. https://doi. org $/ 10.1080 / 00103628509367600$

Raij, B. (2011). Fertilidade do solo e manejo de nutrientes. International Plant Nutrition Institute.

Raij, B., Cantarella, H., Camargo, A.P., \& Soares, E. (1982). Perdas de cálcio e magnésio durante cinco años em ensaio de calagem. Revista Brasileira de Ciência do Solo, 6,33-37.http://www.embracal.com.br/info-agrico/ textos-completos/texto\%2021.pdf

Rodrigues, L.A., Martinez, H.E.P., Neves, J.C., Novais, R.F., \& Mendonça, S.M (2001). Growth response of coffee tree shoots and roots to subsurface liming. Plant and Soil, 234(2), 207-214. https://doi. org/10.1023/A:1017999318532

Rodrigues, L.A., Martinez, H.E., Neves, J.C., Novais, R., \& Mendonça, S. M. (2006). Respostas nutricionais de cafeeiros Catuaí e Icatu a doses de calcário em subsuperficie. Revista Brasileira de Ciência do Solo, 30(6), 985-995. https://doi.org/10.1590/S010006832006000600008

Sadeghian, S. (2003). Efecto de la fertilización con nitrógeno fósforo, potasio y magnesio sobre las propiedades químicas de suelo cultivados en café. Revista Cenicafé, 54(3), 242-257. http://hdl.handle.net/10778/273

Sadeghian, S. (2010). La materia orgánica: Componente esencial en la sostenibilidad de los agroecosistemas cafeteros. Cenicafé. http://hdl.handle.net/10778/1113

Sadeghian, S. (2013). Nutrición de cafetales. En Federación Nacional de Cafeteros de Colombia (Ed.), Manual del cafetero colombiano: Investigación y tecnología para la sostenibilidad de la caficultura (Vol. 2, pp. 85-116). Cenicafé.

Sadeghian, S. (2016). La acidez del suelo una limitante común para la producción de café. Avances Técnicos Cenicafé, 466, 1-8. http://hdl.handle.net/10778/704

Sadeghian, S., \& Zapata, R. (2012). Propiedades relacionadas con la adsorción de cationes intercambiables en algunos suelos de la zona cafetera de Colombia. Revista Cenicafé, 63(2), 79-89. http://hdl.handle.net/10778/537

Silva, I., \& Mendonça, E. (2007). Matéria orgânica do solo. En R. Novais, V. Álvarez, N. Barros, R. Fontes, R. Cantarutti, \& J. Neves (Eds.), Fertilidade do solo(1ra ed., pp. 275-374). Sociedade Brasileira de Ciência do Solo. 
Soratto, R., \& Crusciol, C. A. (2008). Métodos de determinação de cálcio e magnésio trocáveis e estimativa do calcário residual em um Latossolo submetido à aplicação de calcário e gesso em superfície. Revista Brasileira de Ciência do Solo, 32(2), 663-673. http:// www.scielo.br/pdf/rbcs/v32n2/21.pdf

Sousa, D., Miranda, L., \& Oliveira, S. (2007). Acidez do solo e sua correção. En R. Ferreira-Novais, V. Álvarez, N. Barros, R. Fontes, R. Cantarutti, \& J. Lima (Eds.), Fertilidade do solo (1ra ed., pp. 205-274). Sociedade Brasileira de Ciência do Solo.

Suárez, F., \& Rodríguez, A. (1956). Aplicación de la cal en cafetos jóvenes. Revista Cafetera de Colombia, 12(129), 4294-4301. http://hdl.handle.net/10778/1108

Valencia, G., \& Bravo, E. (1981). Influencia del encalamiento en la producción de cafetales establecidos. Revista Cenicafé, 32(1), 3-14. http:// hdl.handle.net/10778/1109
Vilela, L.C., Santos, A.C., Barreto, P.M., Brito, S., Silva, J.E.C., \& Oliveira,L.B.T. (2010). Propriedades químicas de Latossolo Vermelho em função da aplicação de gessageme calagem. Revista Acadêmica CiênciaAnimal, 8(1), 19-27. https://doi.org/10.7213/cienciaanimal. v8i1.10385

Vinasco, C., Valencia, G., \& Chaves, B. (1992). Cambios químicos en suelos incubados con fuentes y dosis de calcio. Revista Cenicafé, 43(1), 5-13. http://hdl.handle. net/10778/1112

Wagatsuma, T., \& Ezoe, Y. (1985). Effect of pH on ionic species of aluminum in medium and on aluminum toxicity under solution culture. Soil Science and Plant Nutrition, 31(4), 547-561. https://doi.org/10.1080/003 80768.1985.10557463

Zapata, R.D. (2004). Química de la acidez del suelo. Cargraphics. 